\title{
Repeated self-injury from a liaison psychiatry perspective
}

\author{
Mark Broadhurst \& Paul Gill
}

Abstract Self-injury (self-harm) occurs frequently and is familiar in the accident and emergency departments and surgical and medical wards of 'acute hospitals'. Despite its common presentation, there are frequent and authoritative reports that patients' experiences of emergency hospital treatment for self-harm are often negative. There is compelling evidence for the need to improve delivery of care for patients who self-harm, and to improve the training, supervision, support and coordination of the staff groups delivering that care.

This article joins two recent submissions to APT on the subject of self-injury, by Leonard Fagin (Repeated self-injury: perspectives from general psychiatry, (2006), 12, 193-201) and Jack Nathan (Self-harm: a strategy for survival and nodal point of change, (2006), 12, 329-337.

The assessment and management of people who repeatedly self-injure (self-harm) has always been a major part of the work of psychiatrists. It is increasingly clear, however, that this process is complicated by two specific issues - the reluctance of a significant proportion of these patients to present to an accident and emergency (A\&E) department for medical treatment and psychiatrists' limited ability to predict repetition of the act. Although the focus of this article is people who present themselves to 'acute hospitals' for medical treatment following self-injury, it is important to remember that the majority of people who self-injure do not go to hospital (Hawton et al, 2002; Meltzer et al, 2002). For those who do, the A\&E department is usually their first point of contact.

About $80 \%$ of people who go to A\&E following an act of self-harm have taken an overdose, even though behaviours such as cutting may be twice as prevalent as overdose in those who self-harm in the community (Horrocks et al, 2003).

There are undoubtedly many reasons for not seeking hospital treatment after self-injury, but one important factor is previous poor experience in an A\&E department. The National Institute for Health and Clinical Excellence (NICE) has noted that attendance at $A \& E$ is frequently a negative experience for people who have harmed themselves, and this is likely to influence future acts and attitudes (National Institute for Health and Clinical Excellence, 2004).

Self-harm is a major risk factor for future suicide: around one-quarter of suicides are preceded by acts of self-harm within the previous year (Owens \& House, 1994). It therefore follows that peoples' experience of A\&E attendance has significant safety implications. A bad experience that results in the feeling that A\&E is unhelpful (or worse) may stop an individual from seeking emergency help again, and thus may be indirectly associated with a death years later. Of course, these considerations apply not only to A\&E but to the patient's journey through services in their entirety, and clearly an off-putting experience at any point in the journey may cause future avoidance. Conversely, a previous good experience of services is likely to improve future engagement and cooperation with management plans.

In this article we consider aspects of a patient's journey through A\&E departments and surgical wards, staff attitudes and the responsibilities of professional staff in relation to people who have harmed themselves.

\section{The A\&E department}

In the UK, care in an A\&E department for self-harm patients may be divided into three main components. An initial assessment of the individual allows informed decisions about urgency and the type

\footnotetext{
At the time of writing, Mark Broadhurst was a specialist registrar in liaison psychiatry in Sheffield. He is currently working as a consultant in general adult psychiatry in Derbyshire, where he intends to maintain his interest in liaison psychiatry. Paul Gill has been a consultant in liaison psychiatry, working with the Sheffield Care Trust (The Longley Centre, Norwood Grange Drive, Sheffield S5 7JT, UK. Email: paul.gill@sct.nhs.uk), for the past 9 years. He previously worked for 10 years as a consultant in general adult psychiatry with special interest in liaison psychiatry.
} 
of referral within the department. Next, any acute physical problems arising from the act of self-harm are managed. Finally, the individual is referred for a psychosocial assessment, which is usually carried out by a member of a mental health crisis team or a psychiatric trainee on call.

\section{Initial assessment}

It is common for A\&E departments to use a triage process, whereby the patient's presenting complaint is assessed by an experienced A\&E nurse, who decides how urgently the patient needs to be seen by a casualty officer. Many traditional triage protocols have a tendency to concentrate on physical rather than mental health risk. This allows an element of subjectivity, which means that prioritisation of mental health issues is likely to vary between departments and practitioners. Some triage protocols, such as one originating in Sydney, Australia, include a mental health component.

Many A\&E departments are replacing the triage system with the 'greet and treat' system, whereby the nurse greeting the patient takes responsibility for that person's subsequent care.

One problem with both systems is that the hierarchy of need may become confused with a hierarchy of deservedness; most psychiatrists will have heard

\section{Box 1 Psychosocial assessment}

- The NICE self-harm guidelines recommend that psychosocial assessment be offered to all who have self-harmed

- The process should be an assessment of need as well as risk

- Assessment findings should be used to inform management of the individual's illness

- In current practice, the likelihood of discharge from A\&E to home makes comprehensive assessment invaluable

- Assessment should take place in a timely fashion

- Assessment should continue throughout the patient's hospital stay and all professionals should be involved

- The patient's privacy and right to support during the interview should be respected

- The management plan following the assessment should be communicated to all professionals involved in treatment

- Training improves staff's assessment skills, which in turn reduces risk of patient's future self-harm and suicide comments regarding patients who have self-injured such as 'He can wait - it's his own fault anyway'.

\section{Physical care}

The role of the staff in A\&E is to assess the patient's physical state, address immediately life-threatening problems, determine whether further physical interventions are necessary, link the patient to further care, and make an initial assessment of the patient's mental state and risk factors.

The Department of Health's (2004) requirement that people in A\&E departments be treated within $4 \mathrm{~h}$ of arrival has benefited patients because they are not left for many hours unattended, but it has also added a sense of pressure and need to hurry that they often feel. This pressure also bears on staff, and when they have to depend on colleagues outside of the A\&E department (who have different targets to meet) to carry out mental health assessments it is not surprising that the 'outsiders' face an angry response if they cannot meet A\&E's time target. Resulting conflict between mental health and A\&E staff may cause the patient to experience assessments in less than ideal circumstances. Such conflict may also sow in the minds of A\&E staff the seed for a lack of confidence in the response of the mental health team, which is likely to lead to increased anxiety about mental health patients who present to A\&E. It is also possible that the threshold for admission to the acute hospital may be lowered to avoid breaches of the $4 \mathrm{~h}$ wait.

\section{Psychosocial assessment}

The importance of psychosocial assessment (Box 1) has been highlighted by Kapur et al (1998) in their study of the management of self-harm patients in hospital. They found evidence suggesting that, because of bed cuts, general hospital staff are avoiding admitting patients thought to be at low physical risk and that admission to psychiatric beds is largely reserved for those with severe mental illness. People who have self-harmed tend to fall between these two categories, being deemed neither at high physical risk nor to have a severe mental illness. Moreover, many were discharged from A\&E with no psychosocial assessment at all.

The timing of assessment

The NICE guideline on self-harm (National Institute for Health and Clinical Excellence, 2004) states that everyone who has self-harmed should be offered a psychosocial assessment. For those who have presented to $A \& E$ and are not to be admitted to a ward for medical care, the assessment should 
be offered before discharge (some services offer the patient an appointment for the next day). If the patient is to be admitted for acute medical treatment, the psychosocial assessment is usually conducted on the ward. Kapur (2005) stresses the point that psychosocial assessment should be an assessment of need, rather than simply an assessment of risk.

\section{Who should conduct the assessment?}

There are several reasons why initial psychosocial assessment of people who present to A\&E with selfharm should be carried out by general hospital staff. First, not all individuals who self-injure agree to see mental health staff and this is not always predictable at the time of admission. Second, people who have self-harmed frequently complain that A\&E staff are not interested in them or their problems: a psychosocial assessment may indicate to them that this is not the case.

Psychosocial assessment should be conducted by a member of staff who has been trained in the process and who has access to regular supervision, which is probably best provided by mental health staff. The assessment should take place in privacy. The interviewer should also offer the patient the option of including a relative or friend for part (but not all) of the interview. At the end of the assessment, the decision reached regarding further care should be communicated to the A\&E staff, preferably with reasons for it. The responsibility of facilitating assessment by referring patients who are fit to be assessed and providing an appropriate room and privacy in which to conduct the assessment falls to the hospital running the A\&E department.

There are anecdotal reports from many A\&E departments and from mental health services that the introduction of mental health emergency teams in England and Wales (and, to a lesser extent, other parts of the UK) has reduced the availability of psychosocial assessment in A\&E departments. This is because a number of these teams have set criteria for accepting patients that exclude many individuals requiring this type of assessment in A\&E. The differing targets determined centrally for A\&E departments and mental health emergency teams do not necessarily make a comfortable fit.

The assessment of risk, and of the patient's mental state, should be an ongoing process while the patient is in the hospital. It is therefore a responsibility shared by acute hospital staff as well as mental health service staff.

\section{Staff training}

The importance of training in psychosocial assessment cannot be overstated. Melville \& House (1999) showed that inadequate training results in inade- quate care, because of incomplete documentation of the assessment and of the patient's clinical needs. This is likely to lead to the patient's dissatisfaction and ultimately result in avoidance of health services (Dennis et al, 1990; Ryan et al, 1998). Indeed, there is evidence that incomplete assessment increases the risk of repetition of self-harm threefold (Crawford \& Wessely, 1998), with a consequent increase in the risk of suicide.

\section{The surgical ward}

If self-injury results in serious damage to tendons, vessels or other structures, admission to a surgical ward, and possible elective surgery, is often indicated. Surgical wards are not uniform and not all specialties have the same degree of experience of patients who have injured themselves. For example, plastic surgery and general surgical wards tend to treat a large number of people after self-injury, whereas ear, nose and throat or maxillofacial wards see far fewer. This may affect staff attitudes and patients' experiences on the ward: greater exposure to patients who self-harm can mean that ward procedures are more geared to their needs; on the other hand, familiarity may lead to a somewhat dismissive approach.

\section{Fitting in on the ward}

Owing to the nature of many surgical conditions and treatments, surgical patients are often largely confined to their bed space and are significantly limited in their activity. Ambulant patients on a surgical ward have usually gone through a period of illness and inactivity before becoming well enough to walk around, and have generally become habituated to the rhythms of ward life. However, people who have self-harmed are often ambulant but will not have become habituated to the surgical ward, and ward staff are often uncertain how to respond to such a situation. As a result, self-injury patients on surgical wards frequently complain of long periods of inactivity and boredom, or that they are being unnecessarily restricted. Restrictions imposed by ward regimes cause many to feel that they have had to hand control to the ward staff.

\section{Analgesia}

A common problem is that of analgesia. There is evidence that self-harm patients are offered analgesia less frequently than others. This has been confirmed by patient interviews carried out in the preparation of the NICE guideline on self-harm: many said 
that they were offered no or inadequate analgesia following self-injury (National Institute for Health and Clinical Excellence, 2004: p. 81). Two specific quotes give pause for thought:

'I said it hurts. They said, “Well, it didn't hurt when you cut it".'

and

'Obviously you enjoy the pain, you know so so [sic] maybe you need stitching up without it.'

A particular source of conflict arises in the case of opiate users, because of the incorrect assumption that a patient's routine opiate requirement alone will provide effective analgesia.

\section{Low self-esteem}

A high proportion of people who harm themselves have low self-esteem (Favazza, 1989), and conditions that ward staff view as normal may exacerbate the problems of such patients (Pembroke, 1991). Anumber of factors might reinforce the negative cognitions of patients with mental health problems.

First, surgical wards are often characterised by a sense of remote efficiency, which may be misinterpreted by someone with low self-esteem as disapproval.

Second, lack of privacy - both because of limited availability and lack of understanding about its necessity-means that patients who have self-injured may be reluctant to discuss their problems with staff looking after them. The 'public' nature of the surgical ward round can embarrass patients and make them feel that their problems are being aired for all to hear. Again this may lead to a reluctance to be candid with the team.

Finally, decisions about discharge are frequently made during the ward round and acted on immediately. This may come as a surprise to, and be a source of distress for, patients, carers and even the mental health team, who are all accustomed to the slower pace and greater planning associated with discharge from psychiatric wards.

The patient's journey through the surgical ward may therefore be fraught with stressors, and these may well be compounded by the swift pace of the surgical out-patient clinic.

\section{Patients' experiences}

A patient's experience of contact with medical services may be crucial to their well-being and future help-seeking behaviour. McAllister et al (2002), drawing on numerous studies exploring the personal experiences of those who have self-harmed, found that many felt they were ignored, made to wait, given painful treatments and being judged and punished by staff. It has been reported that even unconscious negative attitudes towards self-harm can be revealed in staff's demeanour and manner, leading patients to sense rejection, which may in turn prompt further self-harm (Hemmings, 1999). It is therefore important to consider the effect that such contact is likely to have on the patient.

Attending A\&E, being admitted to a surgical ward and attending out-patient clinics all involve relinquishing of control to strangers - a process that would cause a sense of discomfort in most of us. For patients presenting with repeated self-injury, in whom issues of control are already a major difficulty, ceding control to others can be very frightening indeed.

A few find that handing over control in this way comes as a welcome relief and it is not unheard of to hear a self-harm patient admitted to a hospital ward say 'I haven't felt so well for years'. Although this response occurs infrequently, it is important to recognise it: it is possible that it will serve as positive reinforcement of self-harm, as the same outcome may be sought in future crises, thus perpetuating acts of self-injury that will result in admission. Much more frequently it is patients' pre-existing low self-esteem that is reinforced by their contact with services. It is also possible that certain patients will specifically seek out the unpleasant process of relinquishing control as part of a need for self-punishment.

\section{Frequent attenders}

Frequent attendance at hospital because of selfinjury is more usually an issue for A\&E departments than surgical wards. However, such patients present challenges for all staff. In particular, staff may react with higher levels of hostility towards them. Also, familiarity with a frequent attender may result in reduced communication and, specifically, in a failure to recognise new problems. The pathway of care may become repetitive, with repeated assessments coming to the same conclusion, a result that can become irritating for staff and patient alike. A further difficulty is the issue of splitting. Individual staff members may identify with different aspects of the patient's difficulties or psychological state (Huband \& Tantam, 2000). Contrasting identification processes in different staff members may then lead to conflict between staff, usually to the detriment of the patient's care.

Although increased hostility towards frequent attenders is the most common problem, occasionally a frequent attender will elicit inappropriate levels of identification in some staff members. 


\section{Coordinated care and care plans}

The treatment of people who frequently go to A\&E departments following self-injury is an example of the desirability of fostering coordinated and goodquality working across a number of services.

One important issue is that the patient (in the UK) should at least be considered for the enhanced care programme. Should enhanced care be inappropriate, a plan should be drawn up covering what to do in the event of further attendance following self-injury. This plan should be agreed between the patient, relatives or carers, acute staff, mental health staff and (where appropriate) ambulance service staff. It should state the level of assessment deemed appropriate after future episodes and should specify who should be informed of a repeated incident. The plan should be available to staff in the A\&E department, to the patient and to mental health staff. An inferred element of such a care plan is that it should be practical, and that professionals are able, and willing, to fulfil their part in it. The responsibility falls to the professionals involved to ensure that they work together to give the plan the best chance of success.

\section{Staff attitudes}

In the preceding section we considered the patient's experience of contact with services and the ways in which this affects future contact. Clearly, the attitudes of staff caring for the patient are intricately entwined with this and should also be addressed.

It has been noted that staff responses to people who have self-injured by, for example, cutting or burning are frequently less sympathetic than to those who have overdosed, possibly because the perception of overdose as a serious suicide attempt is seen to legitimise the individual's need for care (Clarke \& Whittaker, 1998). It is interesting to note that staff often experience a sense of loss of control similar to that felt by patients. This often accompanies a feeling of inability to cope with a clinical situation (Simpson, 1980). This finding may be helpful in thinking about the negative attitudes of staff towards this patient group.

A further source of tension may be the differing expectations of the staff and patient. The role of the staff in A\&E or on a surgical ward is to address the immediate physical problem and discharge the patient as soon as possible. This may also be the expectation of the patient, but in many cases the patient has a number of other problems to face that this will not resolve.

It is likely that hospital staff's attitudes towards patients who repeatedly self-injure are in part related to the quality of the working relationship between the staff and the mental health team and their confidence in one another. It is therefore important to foster good working relationships, and this has to be clearly seen as the dual responsibility of both sides.

There is little doubt that patients who self-injure gain the impression that staff see them as less deserving than other patients (in extreme situations staff have overtly expressed this). As this is often the patients' own view of themselves, such attitudes by the staff caring for them will only reinforce this belief and exacerbate their difficulties.

The training of staff is very important in the formation of their attitudes towards different patient groups. In our opinion, negative training experiences in dealing with people who have self-harmed often result in the development of negative attitudes towards these patients that can remain with staff throughout their career. By transmission of these attitudes from trainers to trainees, our current career structure could be said to foster negative attitudes. In the past, the tendency to leave the treatment of people who have self-harmed to the most junior members of staff has reinforced the message that this is a low-priority group of patients.

\section{Changing attitudes}

The NICE self-harm guidelineidentifies staff attitudes as a crucial area for improvement in service delivery. We suggest that improvement in attitudes will require a variety of processes, including improved training for both acute hospital and mental health service staff, with the involvement of service users. Ideally, service user involvement would be aided by the development of ongoing evaluation of their experiences and views. Improved collaboration between acute hospitals and mental health services could lead to increased understanding of the problems and needs of this patient group, with a resultant change in staff attitudes and, as a further consequence, improved confidence between acute hospitals and mental health services.

However, a number of barriers to achieving these improvements exist. Most importantly, self-harm is seen as a low priority by everybody in both acute hospitals and mental health services. Furthermore, during training many now senior clinicians will have seen consistently poor outcomes for people presenting to A\&E after self-harm; therefore they too prioritise other patients. This process of fostering negative attitudes may therefore continue until better support is provided for staff members who provide services to people who self-harm. Interestingly, because the needs of people who self-harm are the responsibility of several different elements in the health service, there can be a tendency to see 
it as 'someone else's problem'. For the same reason, there can also be a tendency to blame another part of the service for perceived deficiencies, leading to the assumption that responsibility for change lies elsewhere.

\section{Management of self-harm}

Kapur (2005) reminds us that, despite very extensive literature review in preparation for the NICE self-harm guideline, the evidence that exists is, in fact, relatively limited. Drawing definitive conclusions about management when individuals with this complex clinical problem present to A\&E is therefore difficult. As mentioned above, one of the major points highlighted by the NICE guideline appears to be the shift from assessment of risk to the psychosocial assessment of need. The value of suicide risk assessment is difficult to quantify, as the outcome is rare. Psychosocial assessment, considering psychological and social factors that may have precipitated self-harm, can give rise to a well-informed management plan that attempts to address stressors in these areas. Kapur suggests a strategy whereby all patients who self-harm receive a basic intervention, and those identified as at high risk on the basis of psychosocial and risk assessment receive more intensive support.

\section{Role of mental health staff}

Clearly, management of self-injury on presentation to an A\&E department requires input from both medical and mental health staff. The latter have a particular role in assisting the medical staff in the provision of optimum care by advising in a number of areas (Box 3). Their expertise in the psychosocial assessment has already been discussed, and this assessment should direct them in their collaboration with patients on managing psychosocial stressors. Mental health staff should be key players in arranging well-planned and supported discharge, and ensuring appropriate follow-up aimed at management of future risk and adjustment of the plan as necessary to address new needs. Their input should be timely and lead to prompt action to deliver the management plan.

There has in the past been a tendency for decisions taken by mental health professionals to be misunderstood by their medical colleagues in the acute hospital. An example might be the contrast between a frequent attender with a history of serial self-harm who is discharged from hospital and a quiet person who has never previously harmed themselves who is immediately admitted to an acute psychiatric ward. Although from a mental health perspective this may make perfect sense, from the perspective of the medical staff it may appear inexplicable. Mental health staff have some responsibility to explain their decisions; this will help their colleagues to understanding the issues involved and assist in the promotion of mutual confidence between staff groups.

In addition, mental health staff may have special and frequent experience of psychiatric presentations of physical illness that are sometimes not recognised by medical staff in an acute hospital, and it is clearly their responsibility to consider such diagnoses and draw them to the attention of the medical staff.

Fulfilment of these functions can in itself teach medical staff in acute hospitals how to deal with a patient who repeatedly self-injures. However, perhaps one of the most important roles of mental health staff in the acute hospital is to deliver organised training sessions to address the issues discussed here, to increase their familiarity with the problems of selfharm patients and improve relationships between mental health and medical staff.

\section{Conclusions}

People who have harmed themselves often face negative attitudes in A\&E departments and on hospital wards, and these tend to reinforce the low self-esteem that is an extremely common in self-injury. Together, the reinforcement of low selfesteem and the negative experiences of hospital attendance may increase future risks. Assessment of risk is therefore crucial in this population and it is the responsibility of all professional staff. However, identifying individuals who will harm themselves again remains a problem: Kapur et al

\section{Box 3 Role of mental health staff}

Mental health staff can help acute hospital staff by:

- advising on capacity and consent issues

- advising on management of behavioural disturbance

- advising on management of mental health problems

- advising on and putting into practice the Mental Health Act

- psychosocial assessment, and appropriate action thereon

- explaining psychiatric decisions to them

- arranging prompt discharge to the appropriate service

- identifying unrecognised physical disorder

- training 
(2005) showed that the majority of episodes of repeat self-harm occurred in those assessed as being at low or moderate risk of repetition. This implies that targeting interventions at those rated as high risk may not have a major beneficial effect.

Assessment of psychosocial need should be offered to all patients who self-injure, although healthcare providers still have difficulty in delivering this (Bennewith et al, 2004).

Finally, to improve the assessment and care of those who present for emergency medical treatment for self-harm there is a need for better working relationships between staff groups in the acute hospital, as well as for more structured training and improved mutual trust.

\section{Declaration of interest}

P. G. is a member of the steering group for the Improving Services for People who Self-Harm Project, which has received a grant from the Health Foundation.

\section{References}

Bennewith, O., Gunnell, D., Peters, T., et al (2004) Variations in the hospital management of self-harm in adults in England: observational study. BMJ, 328, 1108-1109. doi:10.1136/ bmj.328.7448.1108.

Clarke, L. \& Whittaker, M. (1998) Self-mutilation: culture, contexts and nursing responses. Journal of Clinical Nursing, 7, 129-137.

Crawford, M. \& Wessely, S. (1998) Does initial management affect the rate of repetition of deliberate self-harm? Cohort study. $B M J, 317,985$.

Dennis, M., Owens, D. \& Jones, S. (1990) Epidemiology of deliberate self-poisoning: trends in hospital attendances. Health Trends, 22, 125-126.

Department of Health (2004) 4-hour Checklist: Reducing Delays for AEE Patients. http:/ / www.dh.gov.uk/en/Publicationsand statistics / Publications/PublicationsPolicyAndGuidance/ DH_4085182 (electronic publication only).

Favazza, A. (1989) Why patients mutilate themselves. Hospital and Community Psychiatry, 40, 137-145.

Hawton, K., Rodham, K., Evans, E., et al. (2002) Deliberate selfharm in adolescents: self-report survey in schools in England. BMJ, 325, 1207-1211.

Hemmings, A. (1999) Attitudes to deliberate self-harm among staff in an accident and emergency team. Mental Health Care, 2, 300-302.

Horrocks, J., Price, S., House, A., et al (2003) Self-injury attendances in the accident and emergency department. Clinical database study. British Journal of Psychiatry, 183, 34-39.

Huband, N. \& Tantam, D. (2000) Attitudes to self-injury within a group of mental health staff. British Journal of Medical Psychology, 73, 495-504.

Kapur, N. (2005) Management of self-harm in adults: which way now? British Journal of Psychiatry, 187, 497-499.

Kapur, N,. House, A., Creed, F., et al (1998) Management of deliberate self-poisoning in adults in four teaching hospitals: descriptive study. BMJ, 316, 32-33.

Kapur, N., Cooper, J., Rodway, C., et al (2005) Predicting the risk of repetition after self-harm: cohort study. BMJ, 330, 394-395.

McAllister, M., Creedy, D., Moyle, W., et al (2002) Nurses' attitudes towards clients who self-harm. Journal of Advanced Nursing, 40, 578-586.
Meltzer, H., Harrington, R., Goodman, R., et al (2002) Children and Adolescents Who Try to Harm, Hurt or Kill Themselves. A Report of Further Analysis from the National Survey of the Mental Health of Children and Adolescents in Great Britain in 1999. Office for National Statistics.

Melville, A. \& House, A. (1999) Understanding deliberate selfharm. Nursing Times, 95(7), 46-47.

National Institute for Health and Clinical Excellence (2004) Selfharm: The short-term Physical and Psychological Management and Secondary Prevention of Self-harm in Primary and Secondary Care. Clinical guideline 16. NICE.

Owens, D. \& House, A. (1994) General hospital services for deliberate self-harm. Journal of the Royal College of Physicians of London, 28, 370-371.

Pembroke, L. (1991) Surviving psychiatry. Nursing Times, 87(49), 30-32.

Ryan, M., Parle, M. \& Babidge N. (1998) What precipitates deliberate self-harm? A cognitive behavioural formulation of attempted suicide presentations at an inner city hospital. Australian Health Review, 21, 194-211.

Simpson, M. A. (1980) Self-mutilation. Temple University Press.

\section{MCQs}

1 Accident and emergency:

a attendances resulting in admission to a ward have increased as a result of reduced hospital bed numbers

b staff should avoid any assessment of patients' mental state

c staff should carry out an assessment of risk factors for further self-harm

d triage protocols reliably give appropriate importance to mental health problems

e $4 \mathrm{~h}$ waiting-time limits are always seen as beneficial to patients who have self-injured.

2 Considering self-injury patients on surgical wards:

a all surgical wards have extensive experience of patients who self-harm

b the restrictions placed on patients who have self-injured are always consistent with the level of immobility caused by their injuries

c there is no evidence that patients who self-injure receive less frequent analgesia

d opiate users require less analgesia following self-injury, as they already have sufficient levels of opiate in their system

e patients' low self-esteem may be unintentionally exacerbated by the normal routine of the staff.

3 Patients who attend hospital frequently following self-injury:

a are generally viewed sympathetically and paternalistically by hospital staff

b always, by virtue of familiarity, receive a more thorough assessment

c always require enhanced CPA

d should have a coordinated care plan identifying the kind of assessment that should occur at future attendances

e have complex needs that make any psychosocial assessment by acute hospital staff unnecessary. 
4 The management of self-harm involves:

a risk assessment as an academic fact-finding exercise

b a change from risk assessment to the new concept of psychosocial assessment, which ignores the formulation of a patient's psychological and social problems

c lack of acknowledgement of the cause of harm in order to reduce stress in the patient

d a psychosocial assessment that accurately predicts the risk of repetition

e a management plan informed by thorough psychosocial assessment.

5 The process of changing attitudes to self-injury may be aided by:

a improving training for medical and surgical staff only

b involvement of service users in staff training

c ensuring that acute hospitals and mental health staff work entirely separately of each other

d the continued view that the needs of people who selfharm are someone else's problem

e the tendency to blame other parts of the service for perceived deficiencies.

\section{MCQ answers}

$\begin{array}{lllllll}\text { 1 } & & \text { 2 } & & 3 & 4 & 5 \\ \text { a F } & \text { a F } & \text { a F } & \text { a F } & \text { a F } \\ \text { b F } & \text { b F } & \text { b F } & \text { b F } & \text { b T } \\ \text { c T } & \text { c F } & \text { c F } & \text { c F } & \text { c F } \\ \text { d F } & \text { d F } & \text { d T } & \text { d F } & \text { d F } \\ \text { e F } & \text { e T } & \text { e F } & \text { e T } & \text { e F }\end{array}$

